\title{
A Comparative Study of Periapical Radiographs and Cone-beam Computed Tomography to Detect the Effects of Periapical Lesions
}

\author{
Fatima M Jadu' ${ }^{1}$, Ahmed M Jan ${ }^{2}$
}

\begin{abstract}
Aim: To compare the diagnostic performance of cone-beam computed tomography (CBCT) relative to periapical radiographs (PAs) for detecting the effects of periapical lesions (PLs) on the roots and surrounding structures.

Materials and methods: This prospective study considered teeth with PLs referred for extraction. To be included, the teeth must also have a recent diagnostic quality PA and a recent CBCT examination with the tooth in question imaged clearly. The images were anonymized, scrambled, and reviewed by two calibrated observers. The extracted teeth were sent along with their periapical tissues for histopathologic examination. Data collected included patient age and gender, the tooth in question, and the histopathologic diagnosis of the apical tissues. The observers were required to record the presence or absence of root resorption, encroachment on the maxillary sinus, and compression of the mental foramen and/or mandibular canal.

Results: Cone-beam computed tomography performed superior to PA, detecting more effects on the surrounding structures, but this was only statistically significant for the effect of external root resorption (ERR) $(p<0.001)$. This was true irrespective of the histopathologic diagnosis of PL. Conclusion: The diagnostic performance of CBCT was significantly superior to that of PA for detecting ERR on teeth with PL.

Clinical significance: The diagnostic accuracy of CBCT exceeds that of PA for detecting the effects of PL. However, the diagnostic accuracy is one of many factors to consider when choosing a diagnostic imaging modality for a specific task. Overall, CBCT examinations should be reserved for challenging cases after PAs have failed to provide all the required diagnostic information.

Keywords: Cone-beam computed tomography, Diagnostic accuracy, Periapical lesion, Periapical radiographs, Root resorption.

World Journal of Dentistry (2019): 10.5005/jp-journals-10015-1667
\end{abstract}

\section{INTRODUCTION}

Periapical lesions are considered to be one of the most common conditions that dentists and dental specialists have to manage. Proper management always requires an accurate diagnosis, upon which the management plan is based. Diagnosing PLs fundamentally requires some form of diagnostic imaging. ${ }^{1}$ Periapical radiographs are usually used to detect PLs and to determine their size and effect on the surrounding structures. ${ }^{1}$ These two-dimensional (2D) radiographs are readily available and easy to obtain and interpret. However, their 2D nature precludes the availability of information in the third dimension, which results in superimposition and sometimes limited contrast resolution. ${ }^{1-4}$ Additionally, their small size may limit viewing the entire PL. These limitations of PAs may affect the dentist's ability to appreciate the delicate effects of PLs on their surrounding structures, such as a loss of the lamina dura and resorption of the roots. ${ }^{1}$

Rapidly advancing technology in the diagnostic imaging field has made available three-dimensional (3D) images, such as CBCT, which provides an abundance of diagnostic information without exposing the patient to relatively high radiation doses. The availability of CBCT and its ease of use are also improving. Operators are now able to manipulate and analyze their images with the click of a button. These advancements in imaging technology and image analysis potentially translate to improved diagnostic performance and consequently improved patient care.

The feasibility of CBCT to detect PLs compared to PAs has been extensively studied and reported. However, few studies delved deeper into $\mathrm{CBCT}$ diagnostic performance relative to PAs for PLs
${ }^{1}$ Department of Oral Diagnostic Sciences, Faculty of Dentistry, King Abdulaziz University, Jeddah, Kingdom of Saudi Arabia

${ }^{2}$ Department of Oral and Maxillofacial Surgery, Faculty of Dentistry, King Abdulaziz University, Jeddah, Kingdom of Saudi Arabia

Corresponding Author: Fatima M Jadu, Department of Oral Diagnostic Sciences, Faculty of Dentistry, King Abdulaziz University, Jeddah, Kingdom of Saudi Arabia, Phone: +966 254 00000, e-mail: fjadu@kau. edu.sa

How to cite this article: Jadu FM, Jan AM. A Comparative Study of Periapical Radiographs and Cone-beam Computed Tomography to Detect the Effects of Periapical Lesions. World J Dent 2019;10(5): 346-349.

Source of support: Nil

Conflict of interest: None

and to detect the delicate effects such as root resorption. This is important because it may influence the treatment option or result in treatment modifications. Therefore, the aim of the current study was to compare the diagnostic performance of CBCT relative to PAs to detect the effects of PLs on the roots and surrounding structures.

\section{Materials and Methods}

This prospective study was approved by the research ethics board at the academic institution where the study took place. Teeth referred to the oral and maxillofacial surgery service for extraction due to a periapical lesion were included in the study. There were 
two inclusion criteria: the first was presence of a recent PA of adequate diagnostic quality (density, contrast, no major technical errors) of the tooth prior to extraction and the presence of a recent CBCT examination that includes the tooth in question. Exclusion criteria included primary teeth, impacted teeth, and teeth with incomplete roots. Data collected included the patient's age and gender, the tooth in question, and the histopathologic diagnosis of the periapical lesion after extraction.

Fortunately, all included CBCT examinations were conducted in the same manner using the same iCAT scanner (Imaging Sciences International, Hatfield, PA, USA) and similar exposure parameters (recommended by the manufacture). In addition, all patients were scanned based on the endodontic protocol using a field-of-view (FOV) setting of $16 \mathrm{~cm} \times 6 \mathrm{~cm}$ and a voxel size (VOX) setting of $0.2 \mathrm{~mm}$.

Both sets of images were anonymized, scrambled, and reviewed for the presence or absence of PL effects on the roots and the surrounding structures including the mandibular canal, mental foramen, and maxillary sinus. Two calibrated observers, a certified oral and maxillofacial radiologist and a certified oral and maxillofacial surgeon, reviewed the images. They reviewed the PAs as TIFF images and the CBCT images using Vision software (Imaging Sciences International). The observers were allowed to manipulate the images as they desired, and they were not given any time constraints. However, no more than 10 cases were reviewed per day to avoid fatigue. The calibration process was performed using 10 cases that were not included in the study to ensure uniformity of the terminology and assessment methods. The observers were required to record the presence or absence of root resorption, maxillary sinus encroachment, and/or compression of the mental foramen and/or the mandibular canal.

Extracted teeth and their periapical tissues were placed in $10 \%$ formaldehyde and sent for histopathologic examination.
This study was analyzed using IBM SPSS (SPSS Inc., Chicago IL, USA) version 23. Simple descriptive statistics were used to define the characteristics of the study variables using counts and percentages for the categorical and nominal variables, while continuous variables are presented as the mean and standard deviation. Fisher's exact test was used to establish a relationship between categorical variables. Finally, the criterion to reject the null hypothesis was a conventional $p$ value $<0.05$. Intra- and interobserver reliabilities were assessed using $\mathrm{k}$ statistics.

\section{Results}

Intraobserver reliability ranged from 0.80 to 0.87 (very good agreement) for PAs and 0.72 to 0.80 (good agreement) for CBCT images. Interobserver reliability ranged from 0.66 to 0.82 (good to very good agreement) for PAs and 0.54 to 0.71 (moderate to good agreement) for CBCT images.

Ninety-three patients were included in the study. The patients ranged in age from 19 years to 93 years, with a mean age of 37.4 years. Gender distribution was almost equal (54.8\% females and $45.2 \%$ males). Most cases involved the posterior teeth (86\%), and the mandibular teeth were most commonly involved (59\%).

The $2 \times 2$ tables comparing the performance of the two imaging modalities regarding the three effects (root resorption, effect on the maxillary sinus, and effect on the mental or mandibular canal) are presented in Tables 1 to 3, respectively. The CBCT performed superior to PA, detecting more effects on the surrounding structures, but this was only statistically significant for the effect of root resorption $(p<0.001)$.

Because of a lack of a gold standard (histopathologic confirmation) in the current study, the term "positive percent agreement" was used instead of "sensitivity," the term "negative

Table 1: Comparison between cone-beam computed tomography and periapical radiographs for the effect of root resorption

\begin{tabular}{|c|c|c|c|c|c|}
\hline \multirow{2}{*}{\multicolumn{2}{|c|}{ Root resorption }} & \multirow[b]{2}{*}{ Total } & \multicolumn{2}{|r|}{$P A$} & \multirow[b]{2}{*}{$p$ value } \\
\hline & & & Detected & Not detected & \\
\hline \multirow[t]{2}{*}{ CBCT } & Detected & 67 & 67 (72.0\%) & $5(5.4 \%)$ & $<0.001^{\mathrm{a}}$ \\
\hline & Not detected & 26 & $0(0.0 \%)$ & 21 (22.6\%) & \\
\hline Total & & 93 & 67 (72.0\%) & $26(28.0 \%)$ & \\
\hline
\end{tabular}

aSignificant using Fisher's exact test

Table 2: Comparison between cone-beam computed tomography and periapical radiographs for the effect of encroachment on the maxillary sinus

\begin{tabular}{|c|c|c|c|c|c|}
\hline \multirow{2}{*}{\multicolumn{2}{|c|}{ Maxillary sinus }} & \multirow[b]{2}{*}{ Total } & \multicolumn{2}{|r|}{$P A$} & \multirow[b]{2}{*}{$p$ value } \\
\hline & & & Detected & Not detected & \\
\hline \multirow[t]{2}{*}{ CBCT } & Detected & 3 & $25(61.0 \%)$ & $3(7.3 \%)$ & 0.053 \\
\hline & Not detected & 38 & $0(0.0 \%)$ & $13(31.7 \%)$ & \\
\hline Total & & 41 & 25 (61.0\%) & 16 (39.0\%) & \\
\hline
\end{tabular}

Table 3: Comparison between cone-beam computed tomography and periapical radiographs for the effect of compression of the mental foramen or mandibular canal

\begin{tabular}{|c|c|c|c|c|c|}
\hline \multirow{2}{*}{\multicolumn{2}{|c|}{ Mental/mandibular }} & \multirow[b]{2}{*}{ Total } & \multicolumn{2}{|r|}{$P A$} & \multirow[b]{2}{*}{$p$ value } \\
\hline & & & Detected & Not detected & \\
\hline \multirow[t]{2}{*}{ CBCT } & Detected & 12 & $43(78.2 \%)$ & $1(1.8 \%)$ & 0.218 \\
\hline & Not detected & 43 & $0(0.0 \%)$ & $11(20.0 \%)$ & \\
\hline Total & & 55 & 43 (78.2\%) & $12(21.8 \%)$ & \\
\hline
\end{tabular}


percent agreement" was used instead of "specificity," and the term "overall percent agreement" was used instead of "accuracy." The new terms were calculated in the same manner as the original terms. ${ }^{5}$ The alternate terminology indicates that the estimates are for agreement and not for accuracy. ${ }^{6}$ Table 4 outlines the new estimates and their calculations for the three studied effects of PLs.

The most common histopathologic diagnosis was inflammatory granuloma or radicular cyst (89.1\%). However, 10 cases (10.9\%) were diagnosed with odontogenic cysts or odontogenic tumors. The significant difference in the performance of CBCT compared to PAs for detecting ERR was valid regardless of the histopathologic diagnosis as evident in Tables 5 and 6.

\section{Discussion}

Comparative studies are ideally based on the concept of comparing the questionable concept with the gold standard. However, this was not feasible in the current study and the imaging technique (CBCT) in question was compared to the standard of practice (PAs), with the expectation that CBCT would outperform the standard because it is a 3D imaging modality, while the standard imaging technique is only $2 \mathrm{D}$ and inherently suffers from overlap.

Cone-beam computed tomography performed superior to PAs for detecting the effects of PL. However, ERR was the only effect of PLs that showed a statistically significant difference between the two imaging modalities. Cone-beam computed tomography outperformed PAs in detecting more ERR cases. These results are consistent with those of several other studies. ${ }^{7-10}$ A systematic

Table 4: Positive, negative, and overall percent agreement between periapical radiographs and cone-beam computed tomography images

\begin{tabular}{llll}
\hline & $\begin{array}{l}\text { Positive \% } \\
\text { agreement } \\
\text { (sensitivity) }\end{array}$ & $\begin{array}{l}\text { Negative \% } \\
\text { agreement } \\
\text { (specificity) }\end{array}$ & $\begin{array}{l}\text { Overall \% } \\
\text { agreement } \\
\text { (accuracy) }\end{array}$ \\
\hline Root resorption & 100 & 80 & 94 \\
$\begin{array}{l}\text { Maxillary sinus } \\
\text { encroachment }\end{array}$ & 100 & 81 & 92 \\
$\begin{array}{l}\text { Mental foramen/ } \\
\text { mandibular canal } \\
\text { compression }\end{array}$ & 100 & 91 & 98 \\
\hline
\end{tabular}

review and meta-analysis was conducted in 2017 to compare the accuracy of the two imaging modalities for ERR detection. ${ }^{7}$ The study reviewed only in vitro studies and found that CBCT was more sensitive and more accurate compared to PA. ${ }^{7}$ This was thought to be related to the limited information provided by $P A$, regarding the buccal and lingual root surfaces. ${ }^{7}$ Other studies found that CBCT was more accurate, more sensitive, and more specific than PA for detecting ERR and internal root resorption. ${ }^{8-10}$ Estrela et al. also found that internal root resorption was detected earlier on CBCT images. $^{9}$

A comparison of the diagnostic efficiency of two imaging modalities cannot be considered complete, without a thorough discussion of other factors that should be taken into account, such as radiation dose, cost, and accessibility. With regard to the radiation dose, $\mathrm{CBCT}$ examinations can deliver effective radiation doses ranging from a few $\mu \mathrm{S}_{V}$ to over $1000 \mu \mathrm{S}_{\mathrm{v}}$ depending on many factors including size and centralization of the FOV, the VOX setting, peak kilovoltage $\left(\mathrm{kV}_{\mathrm{p}}\right)$ setting, time setting, and number of basis images. ${ }^{11,12}$ Therefore, it is imperative that dosimetry data are carefully reported and interpreted. In a recent study of impacted canines, CBCT examinations were shown to deliver a dose 15 times greater than a combination of one panoramic radiograph and three PAs. ${ }^{13}$

Two factors are especially important when considering the radiation doses: (1) FOV size and centering and (2) VOX setting. The FOV size is inversely proportional to the effective radiation dose that is delivered to patients. ${ }^{14}$ This seems intuitive because a smaller area of the patient that is exposed to radiation results in a lower radiation dose to the patient. ${ }^{14}$ Equally as important is FOV centralization. Jadu et al. found that CBCT scans centered on the mandible yielded a greater effective radiation dose than those centered on the maxilla. ${ }^{11}$ The proposed explanation was the proximity of the mandibular scans to the thyroid gland, which is considered to be highly radiosensitive. ${ }^{11}$

In the current study, all the CBCT images were made with a VOX setting of $0.2 \mathrm{~mm}$, which is the standard scanning protocol for evaluation of endodontic teeth. A similar VOX setting was chosen by Estrela et al. ${ }^{9}$ Liedke et al. compared the effect of the VOX setting on the ability of CBCT to detect ERR. ${ }^{15}$ They compared three VOX settings $(0.4,0.3$, and $0.2 \mathrm{~mm})$ and found little difference between

Table 5: Comparison between cone-beam computed tomography and periapical radiographs for the effect of root resorption caused by inflammatory periapical lesions

\begin{tabular}{|c|c|c|c|c|c|}
\hline \multirow{2}{*}{\multicolumn{2}{|c|}{ Root resorption }} & \multirow{3}{*}{$\begin{array}{l}\text { Total } \\
21\end{array}$} & \multicolumn{2}{|r|}{$P A$} & \multirow{3}{*}{$\begin{array}{l}p \text { value } \\
<0.001^{\text {a }}\end{array}$} \\
\hline & & & \multirow{2}{*}{$\begin{array}{l}\text { Detected } \\
61(74.4 \%)\end{array}$} & \multirow{2}{*}{$\begin{array}{c}\text { Not detected } \\
3(3.7 \%)\end{array}$} & \\
\hline CBCT & Detected & & & & \\
\hline & Not detected & 61 & $0(0.0 \%)$ & 18 (22.0\%) & \\
\hline Total & & 82 & 61 (74.4\%) & $22(26.0 \%)$ & - \\
\hline
\end{tabular}

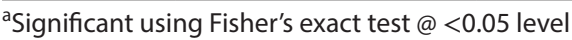

Table 6: Comparison between cone-beam computed tomography and periapical radiographs for the effect of root resorption caused by odontogenic cysts or tumors

\begin{tabular}{|c|c|c|c|c|c|}
\hline \multirow{2}{*}{\multicolumn{2}{|c|}{ Root resorption }} & \multirow[b]{2}{*}{ Total } & \multicolumn{2}{|r|}{$P A$} & \multirow[b]{2}{*}{$p$ value } \\
\hline & & & Detected & Not detected & \\
\hline \multirow[t]{2}{*}{ CBCT } & Detected & 4 & $6(60.0 \%)$ & $1(10.0 \%)$ & $0.033^{a}$ \\
\hline & Not detected & 6 & $0(0.0 \%)$ & $3(30.0 \%)$ & \\
\hline Total & & 10 & $6(60.0 \%)$ & $4(40.0 \%)$ & - \\
\hline
\end{tabular}

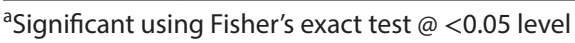


them, but they concluded that the $0.2 \mathrm{~mm}$ and $0.3 \mathrm{~mm}$ VOX settings were better than the $0.4 \mathrm{~mm}$ VOX setting. ${ }^{15}$

External root resorption is an undesirable effect of inflammatory conditions that is caused by clastic cell activation. ${ }^{7,9}$ The condition is progressive but asymptomatic, which means that it can advance undetected clinically. ${ }^{7-9}$ Early detection is crucial because management by performing a root canal treatment can arrest the resorptive process and improve the treatment outcome. ${ }^{7,15}$ The burden of detecting ERR falls on diagnostic imaging. ${ }^{7}$ Despite consistent results confirming the superiority of $\mathrm{CBCT}$ at detecting root resorption compared to $\mathrm{PA}$, the general consensus is that it should be reserved for special cases and should not be considered as the standard of practice for detecting ERR or internal root resorption. ${ }^{8,16}$ There is also a general consensus that every effort to reduce the radiation dose to the patient, starting with the application of patient selection criteria should be made for every patient. ${ }^{16}$

Future studies should address in more detail the influence of the PL size and its location on its detectability using the two imaging techniques. Another future direction should attempt to identify patients who would benefit from $\mathrm{CBCT}$ examinations to simplify the process for dentists and dental specialists.

\section{Conclusion}

The diagnostic performance of CBCT was significantly superior to PAs for detecting ERR on teeth with PLs.

\section{Clinical Significance}

The diagnostic accuracy of $\mathrm{CBCT}$ exceeds that of PA for detecting the effects of PL. However, the diagnostic accuracy is one of many factors to consider when choosing a diagnostic imaging modality for a specific task. Overall, CBCT examinations should be reserved for challenging cases, especially when PAs fail to provide all required diagnostic information.

\section{References}

1. Balasundaram A, Shah P, Hoen MM, et al. Comparison of cone-beam computed tomography and periapical radiography in predicting treatment decision for periapical lesions: a clinical study. Int J Dent 2012;2012:920815. DOI: 10.1155/2012/920815.

2. Wallace JA, Nair MK, Colaco MF, et al. A comparative evaluation of the diagnostic efficacy of film and digital sensors for detection of simulated periapical lesions. Oral Surg Oral Med Oral Pathol Oral Radiol Endod 2001;92(1):93-97. DOI: 10.1067/moe.2001.115974.

3. Bender IB. Factors influencing the radiographic appearance of bony lesions. J Endod 1997;23(1):5-14. DOI: 10.1016/S0099-2399(97)80199-9.
4. Halse A, Molven O, Fristad I. Diagnosing periapical lesionsdisagreement and borderline cases. Int Endod J 2002;35(8):703-709. DOI: 10.1046/j.1365-2591.2002.00552.x.

5. Administration SSUSFaD. U. S. Food and Drug Administration. Statistical guidance on reporting results from studies evaluating diagnostic tests. Available from: http://wwwfdagov/MedicalDevices/ DeviceRegulationandGuidance/GuidanceDocuments/ ucm071148htm [Google Scholar]. issued 2007 March 13, cited 2011 December 25.

6. Jadu FM, Lam EW. A comparative study of the diagnostic capabilities of $2 \mathrm{D}$ plain radiograph and $3 \mathrm{D}$ cone beam CT sialography. Dentomaxillofac Radiol 2013;42(1):20110319. DOI: 10.1259/dmfr.20110319.

7. Yi J, Sun Y, Li Y, et al. Cone-beam computed tomography versus periapical radiograph for diagnosing external root resorption: $A$ systematic review and meta-analysis. Angle Orthod 2017;87(2): 328-337. DOI: 10.2319/061916-481.1.

8. Lima TF, Gamba TO, Zaia AA, et al. Evaluation of cone beam computed tomography and periapical radiography in the diagnosis of root resorption. Aust Dent J 2016;61(4):425-431. DOI: 10.1111/adj.12407.

9. Estrela C, Bueno MR, De Alencar AH, et al. Method to evaluate inflammatory root resorption by using cone beam computed tomography. J Endod 2009;35(11):1491-1497. DOI: 10.1016/ j.joen.2009.08.009.

10. Patel S, Wilson R, Dawood A, et al. The detection of periapical pathosis using digital periapical radiography and cone beam computed tomography - part 2: a 1-year post-treatment follow-up. Int Endod J 2012;45(8):711-723. DOI: 10.1111/j.1365-2591.2012.02076.x.

11. Jadu FM, Alzahrani AA, Almutairi MA, et al. The effect of varying cone beam computed tomography image resolution and fieldof-view centralization on effective radiation dose. Saudi Med J 2018;39(5):470-475. DOI: 10.15537/smj.2018.5.21658.

12. Lorenzoni DC, Bolognese AM, Garib DG, et al. Cone-beam computed tomography and radiographs in dentistry: aspects related to radiation dose. Int J Dent 2012;2012:813768. DOI: 10.1155/2012/813768.

13. Kadesjo N, Lynds R, Nilsson M, et al. Radiation dose from X-ray examinations of impacted canines: cone beam CT vs twodimensional imaging. Dentomaxillofac Radiol 2018;47(3):20170305. DOI: 10.1259/dmfr.20170305.

14. Jadu FM, Hill ML, Yaffe MJ, et al. Optimization of exposure parameters for cone beam computed tomography sialography. Dentomaxillofac Radiol 2011;40(6):362-368. DOI: 10.1259/dmfr/81159071.

15. Liedke GS, da Silveira HE, da Silveira $\mathrm{HL}$, et al. Influence of voxel size in the diagnostic ability of cone beam tomography to evaluate simulated external root resorption. J Endod 2009;35(2):233-235. DOI: 10.1016/j.joen.2008.11.005.

16. American Association of $\mathrm{E}$, American Academy of $\mathrm{O}$ and Maxillofacial R. Use of cone-beam computed tomography in endodontics Joint Position Statement of the American Association of Endodontists and the American Academy of Oral and Maxillofacial Radiology. Oral Surg Oral Med Oral Pathol Oral Radiol Endod 2011;111(2):234-237. DOI: 10.1016/j.tripleo.2010.11.012. 